\title{
IDENTIFICAÇÃO DE FUNÇÕES DE TRANSFERÊNCIA DE ORDEM FRACIONÁRIA UTILIZANDO COMO ENTRADA UM DEGRAU
}

\author{
Luiz Antonio Jacyntho*, Marcelo Carvalho Minhoto Teixeira ${ }^{\dagger}$, Edvaldo Assunção ${ }^{\dagger}$, \\ Aparecido Augusto de Carvalho ${ }^{\dagger}$, Renan Fernandes Kozan ${ }^{\dagger}$, Rodrigo Cardim ${ }^{\dagger}$, Marcelo \\ Augusto Assunção Sanches ${ }^{\dagger}$, Marcos Vinicíus Nascimento Junqueira ${ }^{\dagger}$ \\ * UNEMAT - Universidade do Estado de Mato Grosso \\ Departamento de Matemática - Campus de Barra do Bugres \\ Barra do Bugres, Mato Grosso, Brasil \\ $\dagger$ UNESP - Univ Estadual Paulista, Faculdade de Engenharia de Ilha Solteira \\ Departamento de Engenharia Elétrica, Lab. de Pesquisa em Controle \\ Av. José Carlos Rossi, 1370, 15385-000, Ilha Solteira, São Paulo, Brasil
}

\author{
Emails: luizantonioj@unemat.br, marcelo@dee.feis.unesp.br, edvaldo@dee.feis.unesp.br, \\ aac@dee.feis.unesp.br, renankozan@hotmail.com, rcardim@dee.feis.unesp.br, \\ sanches840@yahoo.com.br, marcosvjn@yahoo.com.br
}

\begin{abstract}
This paper proposes a new method for identification of fractional order linear time invariant systems, represented through stable transfer functions. This method was inspired in the procedure presented by (Kosaka et al., 2005) and (Silva et al., 2007) for integer order transfer functions. The difference is in the order of integrators that can be equal to $\alpha$, with $0<\alpha \leq 1$. To validate the method, two identifications were made, the first for the transfer function of fractional order $G(s)=1 /\left(s+s^{0.5}+1\right)$, disregarding their prior knowledge, and the second for transfer function considered in studies of control and evaluation of movements of lower limbs of paraplegic and hemiplegic using electrical stimulation, performed by the Laboratory of Instrumentation and Biomedical Engineering, Department of Electrical Engineering, Faculty of Engineering, UNESP, Ilha Solteira. Finally, we calculated the mean square error of each models output $y_{\alpha}(t)$ found with the output of the transfer function we wish to identify $y(t)$ and was chosen wich had the lowest value. The obtained results showed the good performance of the new procedure.
\end{abstract}

\begin{abstract}
Keywords - Identification systems, Fractional calculus, Fractional order, Transfer function.
Resumo - Neste trabalho é proposto um novo método de identificação de sistemas lineares invariantes no tempo de ordem fracionária, representados através de funções de transferência estáveis. Tal método foi inspirado no procedimento apresentado em (Kosaka et al., 2005) e em (Silva et al., 2007) para funções de transferência de ordem inteira. A diferença está na ordem dos integradores que podem ser iguais a $\alpha$, com $0<\alpha \leq 1$. Para validar o método, foram feitas duas identificações, sendo a primeira para a função de transferência de ordem fracionária $G(s)=1 /\left(s+s^{0.5}+1\right)$, desconsiderando o conhecimento prévio de todos os parâmetros e a segunda para a função de transferência considerada nos estudos de controle e avaliação de movimentos nos membros inferiores de paraplégicos e hemiplégicos através de eletroestimulação, realizados pelo Laboratório de Instrumentação e Engenharia Biomédica, do Departamento de Engenharia Elétrica da Faculdade de Engenharia da UNESP, Campus de Ilha Solteira. Por último, foi calculado o erro médio quadrático da saída de cada modelo $y_{\alpha}(t)$ encontrado com a saída da função de transferência que desejamos identificar $y(t)$ e foi escolhido o que teve o menor valor. Os resultados obtidos confirmam a eficiência do método proposto.
\end{abstract}

Palavras-chave - Identificação de sistemas, Cálculo fracionário, Ordem fracionária, Função de transferência.

\section{Introdução}

Desde a antiguidade, o ser humano tem procurado descrever matematicamente sistemas reais para tentar compreendê-los e a partir daí conseguir encontrar soluções para diversos tipos de problemas relacionados a eles (Aguirre, 2000).

Nos meados dos anos 90, surgiu uma necessidade crescente de obtenção de modelos a partir de dados observados e não exclusivamente partindose das equações que descrevem a física do processo. Assim tornou-se necessária a implementação de uma metodologia para a identificação direta desses modelos dinâmicos oriundos de dados experimentais e uma alternativa que pode ser viável em vários casos, é utilizar a identificação de sistemas, que é uma área de conhecimento que estuda técnicas e alternativas de modelagem que necessitam pouco ou nenhum conhecimento prévio do sistema em questão e também não exige muito tempo para desenvolver um modelo desejado (Aguirre, 2000).

Este trabalho propõe um novo método de identificação de Funções de Transferência (FT) que utiliza uma ferramenta que vem surgindo frequentemente com o passar dos anos, que se chama Cálculo Fracionário $(\mathrm{CF})$ ou cálculo diferencial e integral de ordem não inteira.

Durante aproximadamente três séculos, a teoria do $\mathrm{CF}$ foi desenvolvida principalmente no campo teórico, quase que exclusivamente para os matemáticos, sendo que no campo prático surgiu de um modo mais estruturado apenas em 1974 com o livro escrito por K. B. Oldham e J. Spanier (Oldham and Spanier, 1974), que desempenhou um papel de destaque no desenvolvimento do $\mathrm{CF}$ do ponto de vista de suas aplicações em física, quí- 
mica e em engenharia, surgindo como o primeiro livro inteiramente dedicado a uma apresentação sistemática das idéias, métodos e aplicações do CF (Podlubny, 1999).

Das duas últimas décadas até os dias de hoje, o $\mathrm{CF}$, vem se tornando uma ferramenta muito importante no auxílio da modelagem e também resolução dos mais variados problemas em diversas áreas, tais como, matemática, física, engenharia e pode-se citar um considerável número de pesquisas sobre viscoelástica, processamento de sinais, difusão, modelagem e controle (Machado, 2010) e (Machado et al., 2010). Considerando apenas a identificação de sistemas com o auxílio do CF, pode-se citar os artigos (Deepyaman et al., 2008), (Narang et al., 2010) e (Galvão et al., 2013). O método proposto foi inspirado em (Kosaka et al., 2005) e em (Silva et al., 2007) e pode ser considerado como uma extensão destes trabalhos para o CF também por determinar modelos de ordem fracionária. A relevância deste trabalho é que a sua natureza é bem simples, não necessita de outro tipo de método auxiliar ou ferramenta, é eficiente, de fácil compreensão e pode ser implementado apenas com um programa em Matlab.

\section{Conceitos fundamentais de cálculo fracionário}

Esta seção apresenta os principais conceitos matemáticos do CF abrangendo a definição de integral e as principais definições de derivada fracionária que possibilitam os cálculos necessários para a aplicação do método elaborado neste trabalho.

\subsection{Definição de integral fracionária}

A função Gama $\Gamma(Z)$ desempenha um papel fundamental no CF, pois é uma expressão analítica do fatorial de qualquer número $z$ real, e até mesmo complexo. A sua definição usual é determinada através da integral:

$$
\Gamma(z)=\int_{0}^{+\infty} e^{-t} t^{z-1} d t, \operatorname{Re}(z)>0 .
$$

A necessidade da condição estabelecida na expressão acima, $\operatorname{Re}(z)>0$ quando z é um número complexo, está demonstrada em (Podlubny, 1999). Seja $\alpha \in \Re$ e $\Gamma(\alpha)$ a função Gama, então a fórmula para representar a integral de ordem fracionária $\alpha$ de Riemann-Liouville é denotada por $J^{\alpha}$ :

$$
J^{\alpha} f(t)=\frac{1}{\Gamma(\alpha)} \cdot \int_{0}^{t}(t-\tau)^{\alpha-1} f(\tau) d \tau .
$$

Em relação à derivada de ordem fracionária, existem algumas definições que são utilizadas e vários prós e contras em cada uma delas, mas em resumo a definição de Riemann-Liouville envolve uma inicialização de ordem fracionária, a de Caputo requer condições iniciais de ordem inteira que em vários casos são mais fáceis de aplicar e a de Grünwald-Letnikov é freqüientemente adotada em algoritmos numéricos e controle de sistemas porque inspira um cálculo de tempo discreto, com base na aproximação do tempo (Machado, 2010).

\subsection{Definição de derivada fracionária de Riemann-Liouville}

Seja $f(t)$ uma função diferenciável em um dado intervalo, $\beta$ um número real positivo, $m$ o menor inteiro maior que $\beta$ e $v=m-\beta$. Então a derivada fracionária de $f(t)$ de ordem $\beta$ denotada por $D^{\beta}$ é definida por:

$$
\begin{aligned}
D^{\beta} & f(t)=D^{m}\left[J^{v} f(t)\right] \\
& =\frac{1}{\Gamma(v)} \frac{d^{n}}{d t^{n}} \cdot \int_{0}^{t}(t-\tau)^{v-1} \cdot f(\tau) d \tau, t>0 .
\end{aligned}
$$

\subsection{Definição de derivada fracionária de Caputo}

Sejam $\beta>0, m$ o menor inteiro maior que $\beta$ e $v=m-\beta$, com $0<v \leq 1$.

A derivada fracionária de ordem $\beta$ de $f(t)$ denotada por $D_{*}^{\beta}$ é calculada da seguinte maneira, para valores de $\beta$ compreendidos entre $m-1<\beta<m$ :

$$
\begin{aligned}
D_{*}^{\beta} f(x) & =J^{v}\left[D^{m} f(x)\right] \\
& =\frac{1}{\Gamma(m-\beta)} \cdot \int_{0}^{x} \frac{d^{n} f(\tau) / d \tau^{n}}{(x-\tau)^{(\beta+1-m)}} d \tau .
\end{aligned}
$$

\subsection{Definição de derivada fracionária de} Grünwald-Letnikov

Seja $\alpha$ um número real qualquer, $a$ e $t$, limites inferiores e superiores de derivação respectivamente e $(t-a) / h$ um número inteiro. A derivada fracionária de ordem $\alpha$ de uma função f é igual a:

$$
\begin{aligned}
& D^{\alpha} f(x) \\
= & \lim _{h \rightarrow 0} \frac{1}{h^{\alpha}} \sum_{m=0}^{\frac{t-a}{h}}(-1)^{m} \frac{\Gamma(\alpha+1)}{m ! \Gamma(\alpha-m+1)} f(x-m+h) .
\end{aligned}
$$

\section{Procedimento para identificação de funções de transferência de ordem fracionária}

Nesta seção é proposto um novo método de identificação de FT estáveis e de ordem fracionária, inspirado no procedimento apresentado em (Kosaka et al., 2005) e também na representação do método por diagrama de blocos em (Silva et al., 2007) para FT de ordem inteira.

\subsection{Procedimento para identificação de funções de transferência de ordem fracionária $\alpha(0<$ $\alpha \leq 1)$}

Seja o sistema linear invariante no tempo com uma única entrada representada por $U(s)$, uma 
única saída igual a $Y_{1}(s)=G(s) \cdot U(s), G(s)=$ $\left(b_{\alpha} \cdot s^{\alpha}+b_{0}\right) /\left(a_{\alpha} \cdot s^{\alpha}+a_{0}\right)=b(s) / a(s)$ uma função de transferência, com $b_{\alpha}$ ou $b_{0} \neq 0,0<\alpha \leq 1$ e as seguintes hipóteses para a aplicação do método que são, $G(s)$ é estável, $U(s)=1 / s$ é uma entrada degrau unitário e que $b(s)$ e $a(s)$ são copri$\operatorname{mos}($ isto é não existe $s \in \mathbb{C}$ tal que $b(s)=a(s)=$ $0)$.

Como $G(s)$ por hipótese é estável, tem-se que $a_{0} \neq 0$, daí $G(0)=b_{0} / a_{0}$ e definindo $b_{\alpha}^{\prime}=b_{\alpha} / a_{0}$ e $a_{\alpha}^{\prime}=a_{\alpha} / a_{0}$ obtém-se $G(s)=$ $\left(b_{\alpha}^{\prime} \cdot s^{\alpha}+G(0)\right) /\left(a_{\alpha}^{\prime} \cdot s^{\alpha}+1\right)$, Considerando agora o método de identificação de $G(s)$ representado através do diagrama de blocos na Figura 1, calculando $Y_{1}(s)$, obtém-se:

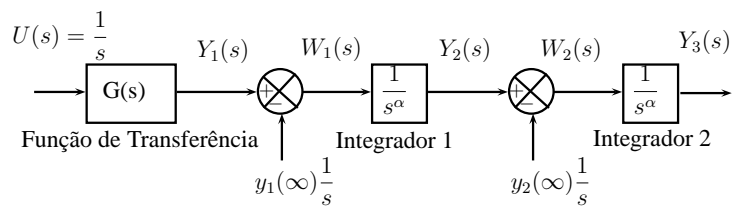

Figura 1: Representação do método através de diagrama de blocos.

$$
Y_{1}(s)=\left[\frac{b_{\alpha}^{\prime} s^{\alpha}+G(0)}{a_{\alpha}^{\prime} s^{\alpha}+1}\right] \frac{1}{s} .
$$

Utilizando o Teorema do Valor Final (TVF), que também é válido para o CF como pode ser verificado em (Deng and Li, 2006), tem-se:

$$
y_{1}(\infty)=\lim _{s \rightarrow 0} s \cdot Y_{1}(s)=G(0) .
$$

Novamente observando a Figura 1, (1) e (2), para o cálculo de $Y_{2}(s)$ conclui-se que:

$$
\begin{aligned}
Y_{2}(s)=\frac{1}{s^{\alpha}}\left[Y_{1}(s)-y_{1}(\infty) \frac{1}{s}\right] \\
=\frac{1}{s}\left\{\frac{1}{s^{\alpha}}\left[\frac{b_{\alpha}^{\prime} s^{\alpha}+y_{1}(\infty)-y_{1}(\infty)\left(a_{\alpha}^{\prime} s^{\alpha}+1\right)}{a_{\alpha}^{\prime} s^{\alpha}+1}\right]\right\} \\
=\frac{1}{s}\left[\frac{b_{\alpha}^{\prime}-y_{1}(\infty) a_{\alpha}^{\prime}}{a_{\alpha}^{\prime} s^{\alpha}+1}\right] .
\end{aligned}
$$

Aplicando novamente o TVF, o resultado fica:

$$
y_{2}(\infty)=\lim _{s \rightarrow 0} s \cdot Y_{2}(s)=b_{\alpha}^{\prime}-y_{1}(\infty) \cdot a_{\alpha}^{\prime} .
$$

Por último, para o cálculo de $Y_{3}(s)$, de acordo com a Figura 1, (3) e (4) o resultado é o seguinte:

$$
\begin{aligned}
Y_{3}(s)=\frac{1}{s^{\alpha}}\left[Y_{2}(s)-y_{2}(\infty) \frac{1}{s}\right] \\
=\frac{1}{s}\left\{\frac{1}{s^{\alpha}}\left[\frac{b_{\alpha}^{\prime}-y_{1}(\infty) a_{\alpha}^{\prime}-y_{2}(\infty)\left(a_{\alpha}^{\prime} s^{\alpha}+1\right)}{a_{\alpha}^{\prime} s^{\alpha}+1}\right]\right\} \\
=\frac{1}{s}\left[\frac{-y_{2}(\infty) a_{\alpha}^{\prime}}{a_{\alpha}^{\prime} s^{\alpha}+1}\right] .
\end{aligned}
$$

Utilizando outra vez o TVF, então :

$$
y_{3}(\infty)=\lim _{s \rightarrow 0} s Y_{3}(s)=-y_{2}(\infty) a_{\alpha}^{\prime} .
$$

Dessa forma, de (4) e de (5) é possível formar um sistema linear de equações do tipo $A \cdot x=b$ :

$A=\left[\begin{array}{ll}1 & -y_{1}(\infty) \\ 0 & -y_{2}(\infty)\end{array}\right], x=\left[\begin{array}{c}b_{2 \alpha}^{\prime} \\ b_{\alpha}^{\prime}\end{array}\right], b=\left[\begin{array}{l}y_{2}(\infty) \\ y_{3}(\infty)\end{array}\right]$

Do sistema linear descrito em (7), supondo $\operatorname{det}(A) \neq 0$, tem-se a solução para $x=A^{-1} . b$. Observe que $\operatorname{det}(A)=-y_{2}(\infty)$ e $\operatorname{de}(4), \operatorname{det}(A)=$ $-y_{2}(\infty)=-\left(b_{\alpha}^{\prime}-y_{1}(\infty) \cdot a_{\alpha}^{\prime}\right) \neq 0$, e isto só irá ocorrer se e somente se o pólo de $\mathrm{G}(\mathrm{s})$ não for igual ao zero de $\mathrm{G}(\mathrm{s})$, conforme demonstração a seguir:

Planta $G(s)=\left(b_{\alpha}^{\prime} s^{\alpha}+y_{1}(\infty)\right) /\left(a_{\alpha}^{\prime} s^{\alpha}+1\right) ;$

Zero de $G(s)$ : igual a s tal que $b_{\alpha}^{\prime} s^{\alpha}+y_{1}(\infty)=0$, ou seja $s^{\alpha}=-y_{1}(\infty) / b_{\alpha}^{\prime} ;$

Polo de $G(s)$ : igual a $s$ tal que $a_{\alpha}^{\prime} s^{\alpha}+1=0$, ou seja $s^{\alpha}=-1 / a_{\alpha}^{\prime}$;

Zero de $G(s)=$ Polo de $G(s)$,

$-y_{1}(\infty) / b_{\alpha}^{\prime}=-1 / a_{\alpha}^{\prime}$;

Portanto, $\left(b_{\alpha}^{\prime}-a_{\alpha}^{\prime} \cdot y_{1}(\infty)\right)=0$.

3.2 Procedimento de identificação de funções de transferência estáveis de ordem $2 \alpha,(0<$ $\alpha \leq 1)$

Seja $G(s)=b_{0} /\left(a_{2 \alpha} \cdot s^{2 \alpha}+a_{\alpha} . s^{\alpha}+a_{0}\right)$, estável, com $b_{o} \neq 0,0 \leq \alpha<1, U(s)=1 / s$ uma única entrada e o diagrama de blocos apresentado na Figura 1. Lembrando novamente que $a_{0} \neq 0$ devido à estabilidade de $\mathrm{G}(\mathrm{s})$, observando que $G(0)=b_{0} / a_{0}$ e definindo $a_{i \alpha}^{\prime}=a_{i \alpha} / a_{0}, i=1,2$, resulta em $G(s)=G(0) /\left(a_{2 \alpha}^{\prime} \cdot s^{2 \alpha}+a_{\alpha}^{\prime} \cdot s^{\alpha}+1\right)$. Agora pela Figura 1 tem-se que:

$$
Y_{1}(s)=G(s) \cdot U(s)=\left(\frac{G(0)}{a_{2 \alpha}^{\prime} s^{2 \alpha}+a_{\alpha}^{\prime} s^{\alpha}+1}\right) \cdot \frac{1}{s} .
$$

Aplicando o TVF, resulta em:

$$
y_{1}(\infty)=\lim _{s \rightarrow 0} s \cdot Y_{1}(s)=G(0)
$$

Observando novamente a Figura 1 para realizar o cálculo de $Y_{2}(s)$, de $(7)$ e de (8), tem-se que :

$$
\begin{aligned}
Y_{2}(s) & =\frac{1}{s^{\alpha}}\left[Y_{1}(s)-y_{1}(\infty) \frac{1}{s}\right] \\
& =\frac{1}{s}\left\{\frac{1}{s^{\alpha}}\left[\frac{y_{1}(\infty)-y_{1}(\infty)\left(a_{2 \alpha}^{\prime} s^{2 \alpha}+a_{\alpha}^{\prime} s^{\alpha}+1\right)}{a_{2 \alpha}^{\prime} s^{2 \alpha}+a_{\alpha}^{\prime} s^{\alpha}+1}\right]\right\} \\
& =\frac{1}{s}\left[\frac{-y_{1}(\infty)\left(a_{2 \alpha}^{\prime} s^{\alpha}+a_{\alpha}^{\prime}\right)}{a_{2 \alpha}^{\prime} s^{2 \alpha}+a_{\alpha}^{\prime} s^{\alpha}+1}\right] .
\end{aligned}
$$

Aplicando o TVF, obtém-se:

$$
y_{2}(\infty)=\lim _{s \rightarrow 0} s . Y_{2}(s)=-y_{1}(\infty) \cdot a_{\alpha}^{\prime}
$$


Por último, para calcular $Y_{3}(s)$ novamente observando a Figura 1, note que de (9) e (10),

$$
\begin{aligned}
Y_{3}(s) & =\frac{1}{s^{\alpha}}\left[Y_{2}(s)-y_{2}(\infty) \frac{1}{s}\right] \\
= & \frac{1}{s}\left\{\frac{1}{s^{\alpha}}\left[\frac{-y_{1}(\infty) a_{2 \alpha}^{\prime} s^{\alpha}-y_{1}(\infty) a_{\alpha}^{\prime}}{a_{2 \alpha}^{\prime} s^{2 \alpha}+a_{\alpha}^{\prime} s^{\alpha}+1}\right]\right\} \\
- & \frac{1}{s}\left\{\frac{1}{s^{\alpha}}\left[\frac{y_{2}(\infty)\left(a_{2 \alpha}^{\prime} s^{2 \alpha}+a_{\alpha}^{\prime} s^{\alpha}+1\right)}{a_{2 \alpha}^{\prime} s^{2 \alpha}+a_{\alpha}^{\prime} s^{\alpha}+1}\right]\right\} \\
= & \frac{1}{s}\left\{\frac{1}{s^{\alpha}}\left[\frac{-y_{1}(\infty) a_{2 \alpha}^{\prime} s^{\alpha}+y_{2}(\infty)}{a_{2 \alpha}^{\prime} s^{2 \alpha}+a_{\alpha}^{\prime} s^{\alpha}+1}\right]\right\} \\
- & \frac{1}{s}\left\{\frac{1}{s^{\alpha}}\left[\frac{y_{2}(\infty)\left(a_{2 \alpha}^{\prime} s^{2 \alpha}+a_{\alpha}^{\prime} s^{\alpha}+1\right)}{a_{2 \alpha}^{\prime} s^{2 \alpha}+a_{\alpha}^{\prime} s^{\alpha}+1}\right]\right\} \\
=\frac{1}{s} & {\left[\frac{-y_{2}(\infty) a_{2 \alpha}^{\prime} s^{\alpha}-y_{2}(\infty) a_{\alpha}^{\prime}-y_{1}(\infty) a_{2 \alpha}^{\prime}}{a_{2 \alpha}^{\prime} s^{2 \alpha}+a_{\alpha}^{\prime} s^{\alpha}+1}\right] . }
\end{aligned}
$$

Aplicando o TVF, chega-se em:

$$
\begin{aligned}
y_{3}(\infty)=\lim _{s \rightarrow 0} s Y_{3}(s) \\
=-y_{2}(\infty) a_{\alpha}^{\prime}-y_{1}(\infty) a_{2 \alpha}^{\prime} .
\end{aligned}
$$

Dessa forma, de (10) e (11) tem-se um sistema linear de equações do tipo $A . x=b$, sendo que:

$$
A=\left[\begin{array}{cc}
0 & -y_{1}(\infty) \\
-y_{1}(\infty) & -y_{2}(\infty)
\end{array}\right], x=\left[\begin{array}{c}
a_{2 \alpha}^{\prime} \\
a_{\alpha}^{\prime}
\end{array}\right], b=\left[\begin{array}{l}
y_{2}(\infty) \\
y_{3}(\infty)
\end{array}\right] \text {. }
$$

Supondo $\operatorname{det}(A) \neq 0$, tem-se a solução para $x=$ $A^{-1}$.b. Observe que $\operatorname{det}(A)=-\left(y_{1}(\infty)\right)^{2}$ e por (8) tem-se que $\operatorname{det}(A)=-\left(y_{1}(\infty)\right)^{2}=-(G(0))^{2}$, mas como $G(0)=b_{0} / a_{0}$ e por hipótese a FT é estável então $a_{0} \neq 0$ e como $b_{0} \neq 0$, sempre existirá solução para o sistema linear descrito em (12).

\section{Identificações realizadas por meio do ambiente Simulink do Matlab}

Para finalizar a última etapa de uma identificação completa, segundo (Landau, 1990) é necessário realizar a validação do modelo. Assim esta seção aborda duas identificações que foram realizadas com a utilização do método proposto neste trabalho e os procedimentos adotados para chegar a esta conclusão.

Covém lembrar que foram fundamentais para o êxito das identificações o auxílio do software Matlab, o ambiente Simulink por proporcionar a construção de um diagrama de blocos do método e o TOOLBOX para Matlab NON-INTEGER utilizado para a simulação do integrador de ordem fracionária $\alpha$ criado por José Sá da Costa Duarte Valério e que gentilmente o colocou à disposição na internet em (Duarte, 2005).

\subsection{Identificação de função de transferência de ordem fracionária}

Para testar o método de identificação desenvolvido em um primeiro teste prático, a in- tenção foi escolher uma FT não muito complexa que apresentasse as características gerais do procedimento da subseção 3.2, então, optouse por $G(s)=1 /\left(1 . s+1 . s^{0,5}+1\right)$. O próximo passo foi escolher os valores de $\alpha$, pois eles podem assumir qualquer valor maior que 0 e menor ou igual a 1 . Assim optou-se por $\alpha=\{0,1 ; 0,2 ; 0,3 ; 0,4 ; 0,5 ; 0,6 ; 0,7 ; 0,8 ; 0,9 ; 1\}$. A partir daí, os coeficientes encontrados com a aplicação do método descrito na Subseção 3.2, para cada $\alpha$ proposto acima são $G(0)=1$ e os seguintes:

Tabela contendo os coeficientes da FT em função do valor de $\alpha$.

\begin{tabular}{|c|c|c|}
\hline Valor de $\alpha$ & $a_{\alpha}$ & $a_{2 \alpha}$ \\
\hline \hline 0,1 & 0,1585 & 0,1407 \\
\hline 0,2 & 0,2512 & 0,2668 \\
\hline 0,3 & 0,3981 & 0,4799 \\
\hline 0,4 & 0,6310 & 0,7763 \\
\hline 0,5 & 1 & 1 \\
\hline 0,6 & 1.585 & 0,3182 \\
\hline 0,7 & 2,512 & $-4,2099$ \\
\hline 0,8 & 3,981 & $-22,3116$ \\
\hline 0,9 & 6,310 & $-84,6839$ \\
\hline 1,0 & 10 & $-281,8$ \\
\hline
\end{tabular}

Como por hipótese a FT deve ser estável, dos modelos de ordem $\alpha$ obtidos com os valores de $\alpha$ escolhidos acima, somente aqueles com ordem $\alpha$ variando entre 0,1 e até 0,6 demonstraram essa característica. Os modelos restantes apresentaram-se instáveis e por este motivo não serão levados em consideração. Na possibilidade de existir modelos estáveis com ordem fracionária entre $0,6 \leq \alpha \leq 0,7$, foi feito um refinamento com os valores de $\alpha=\{0,625 ; 0,650 ; 0,6750\}$ e estão expostos na tabela a seguir.

Tabela contendo os coeficientes da FT em função do valor de $\alpha$.

\begin{tabular}{|c|c|c|}
\hline Valor de $\alpha$ & $a_{\alpha}$ & $a_{2 \alpha}$ \\
\hline \hline 0,625 & 1,7780 & $-0,1767$ \\
\hline 0,650 & 1,9950 & $-1,0320$ \\
\hline 0,675 & 2,2390 & $-2,4169$ \\
\hline
\end{tabular}

Com base nestes dados, é possível concluir que os modelos contendo estes coeficientes são instáveis e por este motivo também serão desconsiderados. Na Figura 2 estão representadas as saídas $y_{\alpha}(t)$ de todas as funções de transferências estáveis $G(s)=b_{0} /\left(a_{2 \alpha} . s^{2 \alpha}+a_{\alpha} . s^{\alpha}+a_{0}\right)$ calculadas acima em resposta a uma entrada degrau unitário. Por último, o critério utilizado para escolher o melhor modelo que irá representar a FT que queremos identificar, foi o erro médio quadrático da saída de cada modelo $y_{\alpha}(t)$ encontrado com a saída da FT que desejamos identificar $y(t)$, cuja a fórmula é a seguinte:

$$
e q m=\sqrt{\sum_{i=1}^{n}\left(y_{i}-y_{\alpha i}\right)^{2} / n}
$$




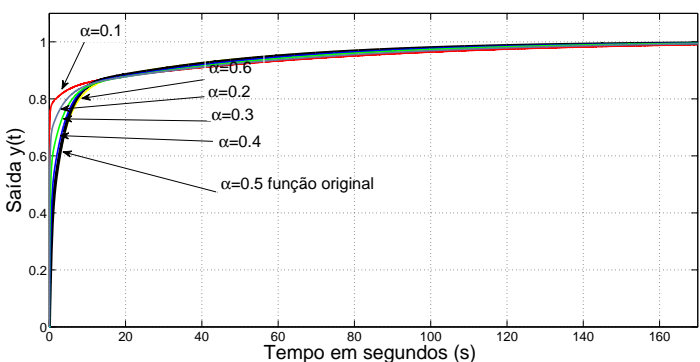

Figura 2: Resposta de todos os modelos estáveis a uma entrada degrau.

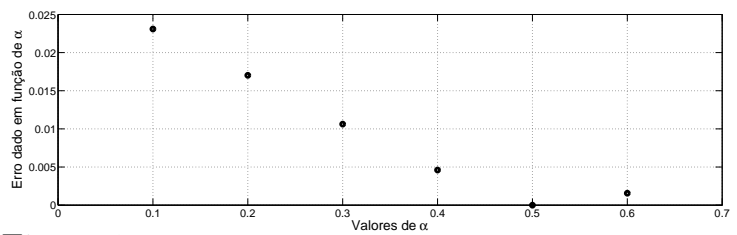

Figura 3: Erro médio quadrátrico em função do valor de $\alpha$.

sendo que $y_{i}=y(i T), y_{\alpha i}=y_{\alpha}(i T), \quad i=$ $1,2, \cdots, n, n=60065$ e $T=0,01665$. Como já foi mencionado que somente nos interessa os modelos matemáticos que apresentaram ser estáveis, somente será realizado o cálculo do erro quadrático destes casos e assim o erro obtido para cada $\alpha, \quad(\alpha, \operatorname{Erro}(\alpha))$ foi: $(0,1 ; 0,0231) ; \quad(0,2 ; 0,0170)$; $(0,3 ; 0,0106) ; \quad(0,4 ; 0,0046) ; \quad(0,5 ; 0) ;(0,6 ; 0,0016)$. Para melhor vizualização, a Figura 3 exibe o gráfico do erro quadrático em função do valor de $\alpha$ adotado.

\subsection{Segunda função de transferência a ser iden- tificada}

Nesta identificação foi levado em consideração um projeto que está sendo desenvolvido no Laboratório de Instrumentação e Engenharia Biomédica do Departamento de Engenharia Elétrica do Campus da UNESP de Ilha Solteira, que realiza Estimulação Elétrica Funcional (FES - Functional Electrical Stimulation) nos membros inferiores de voluntários hemiplégicos e paraplégicos com o objetivo de melhorar a sua qualidade de vida. Neste experimento, estuda-se padrões de estímulos que proporcionem o melhor resultado possível e diante disso é necessário um modelo matemático eficiente para prever o comportamento do músculo. Por este motivo, conforme (Kozan, 2012), foram realizadas simulações com três tipos de modelos e a equipe executora do projeto concluiu que o modelo desenvolvido por (Law and Shields, 2006) foi o mais adequado para representar a FT dos dados coletados de cada paciente, que tem a fórmula $G(s)=b_{0} /\left(a_{2 \alpha} \cdot s^{2 \alpha}+a_{\alpha} \cdot s^{\alpha}+a_{0}\right)$, com $\alpha=1$. Deste modo, para utilizar o método um paciente foi escolhido ao acaso e seus dados utilizados para obter uma FT que neste caso não é conhecida. Escolhendo os valores de $\alpha$ análogo ao exemplo anterior, tem-se $\alpha=$

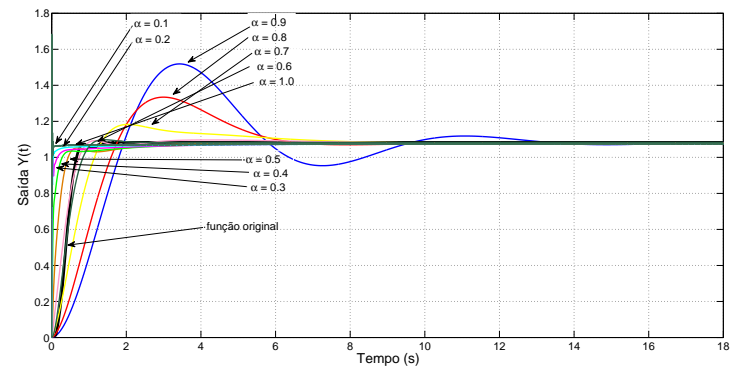

Figura 4: Resposta de todos os modelos calculados a uma entrada degrau.

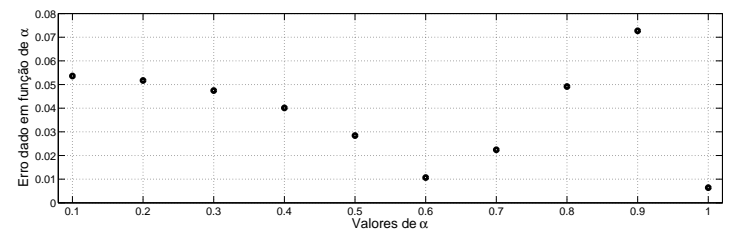

Figura 5: Erro quadrático em função de $\alpha$.

$\{0,1 ; 0,2 ; 0,3 ; 0,4 ; 0,5 ; 0,6 ; 0,7 ; 0,8 ; 0,9 ; 1\}$.

Os coeficientes encontrados para cada $\alpha$ substituído no método descrito na Subseção 3.2 foram os seguintes:

Tabela contendo os coeficientes da FT em função do valor de $\alpha$.

\begin{tabular}{|c|c|c|}
\hline Valor de $\alpha$ & $a_{\alpha}$ & $a_{2 \alpha}$ \\
\hline \hline 0,1 & 0,0266 & 0,0306 \\
\hline 0,2 & 0,0551 & 0,0635 \\
\hline 0,3 & 0,0884 & 0,1011 \\
\hline 0,4 & 0,1271 & 0,1386 \\
\hline 0,5 & 0,1710 & 0,1697 \\
\hline 0,6 & 0,1585 & 0,7648 \\
\hline 0,7 & 0,2194 & 0,1871 \\
\hline 0,8 & 0,3239 & 0,1648 \\
\hline 0,9 & 0,3765 & 0,3089 \\
\hline 1,0 & 0,4271 & 0,0705 \\
\hline
\end{tabular}

Na Figura 4 estão representadas as saídas de todas as FTs calculadas acima em resposta a uma entrada degrau unitário. Conforme foi realizado na identificação anterior, calculando o erro quadrático em função de $\alpha,(\alpha, \operatorname{Erro}(\alpha))$ temse: $\quad(0,1 ; 0,0536) ; \quad(0,2 ; 0,0517) ; \quad(0,3 ; 0,0475)$; $(0,4 ; 0,0401) ; \quad(0,5 ; 0,0204) ;(0,6 ; 0,0107)$; $(0,7 ; 0,0284) ;(0,8 ; 0,0492) ;(0,9 ; 0,0727) ;(1 ; 0,0064)$, utilizando a equação (13), agora com $n=37514$ e $T=0,004$.

A seguir, encontra-se o gráfico do erro quadrático em função de $\alpha$. Como não é possível concluir se o melhor valor de $\alpha$ foi encontrado com os valores escolhidos, será feito um refinamento entre os valores de $\alpha$ compreendidos entre $0,7 \leq \alpha \leq 1$. Deste modo, para os seguintes valores de $\alpha$, o erro quadrático calculado foi o seguinte; $(\alpha,(\operatorname{Erro}(\alpha))$; $(0,975 ; 0,0365) ; \quad(0,950 ; 0,0448) ; \quad(0,925 ; 0,0740)$; $(0,875 ; 0,0418) ; \quad(0,850 ; 0,0382) ; \quad(0,825 ; 0,0346) ;$ $(0,775 ; 0,0262) ;(0,750 ; 0,0222) ;(0,725 ; 0,0180)$.

A seguir, na Figura 5 representa o gráfico do erro quadrático $\operatorname{Erro}(\alpha)$ em função de $\alpha$. Da tabela e do gráfico elaborados com os dados do refinamento, conclui-se que não foi encontrado ne- 


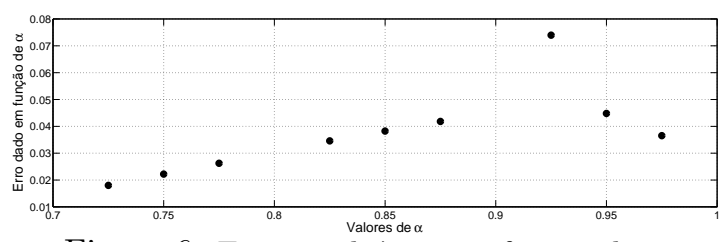

Figura 6: Erro quadrático em função de $\alpha$.

nhum modelo melhor que os calculados anteriormente e diante deste fato o modelo de ordem inteira continuou sendo a melhor representação para a dinâmica do sistema.

\section{Conclusões}

Neste trabalho é proposto um novo método de identificação de FT de ordem fracionária estáveis que necessitam pouco ou nenhum conhecimento prévio de sua natureza. Foram feitas duas identificações, a primeira com a FT $G(s)=$ $1 /\left(1 . s+1 . s^{0,5}+1\right)$ pré estabelecida mas desconsiderando este fato e a segunda com dados coletados de um voluntário, que consiste do deslocamento angular da parte inferior da perna aplicando-se uma eletroestimulação. Na primeira identificação, o modelo fracionário de ordem $\alpha=$ 0,5 obtido coincidiu com a FT original e apresentou erro quadrático igual a 0 . Na segunda identificação, constatou-se que o modelo de ordem inteira igual a 2 apresentou erro quadrático igual a 0,0064 e assim obteve ligeira vantagem sobre o melhor modelo de ordem não inteira $\alpha$ igual a 0,6 , que teve erro quadrático igual a 0,0107. No final, para confirmar a não existência de melhores representações, foi feito um cálculo para valores de $\alpha$ compreendidos entre $0,7<\alpha<1$ e não foi obtido outro modelo com erro quadrático inferior aos já encontrados. Portanto conclui-se que o método é eficaz por ter identificado com precisão a FT de ordem fracionária escolhida no primeiro caso e também que o modelo proposto em (Law and Shields, 2006) que está sendo utlizado no experimento do segundo caso, é melhor que todos os modelos de ordem fracionária obtidos pelo método.

\section{Agradecimentos}

Os autores agradecem o apoio financeiro da CAPES, CNPQ e FAPESP,(Processo n. 2011/17610$0)$.

\section{Referências}

Aguirre, L. A. (2000). Introdução a identificação de sistemas: técnicas lineares e não lineares aplicadas a sistemas reais, 2 edn, UFMG, Belo Horizonte.

Deepyaman, M., Mithun, C. and Amit, K. (2008). A novel approach for complete identification of dynamic fractional order systems using stochastic optimization algorithms and fractional calculus, Proceedings of the 5th International Conference on Electrical and Computer Engineering.
Deng, W. H. and Li, C. P. (2006). Chaos synchronization of the fractional lü system, Open UP University of Pretoria .

Duarte, P. M. O. V. (2005). Toolbox ninteger for matlab. http://web.ist.utl.pt/duarte.valerio/ninteger.

Galvão, R. K. H., Hadjiloucas, S., Kienitz, K. H., Paiva, H. M. and Afonso, R. J. M. (2013). Fractional order modeling of large threedimensional rc networks, IEEE Transactions on Circuits and Systems 60: 624-637.

Kosaka, M., Uda, H., Bamba, E. and Shibata, H. (2005). Dynamic system identification using a step input, Journal of low frequency noyse vibration and active control 24(2): 125 - 134 .

Kozan, R. F. (2012). Controle de posição da perna de pessoas hígidas utilizando um controlador PID, 1 edn, UNESP, ilha Solteira.

Landau, I. D. (1990). System identification and control design using Pim + software cilffs, 1 edn, Prentice Hall, São Paulo.

Law, L. A. F. and Shields, R. K. (2006). Predicting human chronically paralyzed muscle force, Journal of Applied Physiology 100(3): 1027-1036.

Machado, J. A. T. (2010). Optimal tuning of fractional controllers using genetic algorithms, Nonlinear Dyn 2010(62): 447 - 452.

Machado, J. A. T., Silva, M. F., Barbosa, R. S., Jesus, I. S. and Reis, C. M. (2010). Some applications of fractional calculus in engineering, Mathematical Problems in Engineering 2010: 34 p.

Narang, A., Shah, S. L. and Chen, T. (2010). Continuous-time model identification of fractional-order models with time delays, IET Control Theory and Applications 5(9): 900-912.

Oldham, K. B. and Spanier, J. (1974). The fractional calculus, 1 edn, Academic Press, New York - London.

Podlubny, I. (1999). Fractional differential equations, some methods of their solution and some of their applications, 1 edn, Academic Press, San Diego-Boston-New York-London-TokyoToronto.

Silva, D. S., Teixeira, M. C. M., Alvarado, F. V., Assunção, E., Cardim, R. and Gaino, R. (2007). Identificação de funções de transferência utilizando como entrada um degrau, $I$ Simpósio regional de matemática e suas aplicações de Ilha Solteira pp. 40-43. 